\title{
SINGLE IMAGE FOG REMOVAL BASED ON FUSION STRATEGY
}

\author{
V. Thulasika and A. Ramanan \\ Department of Computer Science, Faculty of Science, \\ University of Jaffna, Sri Lanka \\ v.thula.sika@gmail.com, a.ramanan@jfn.ac.lk
}

\begin{abstract}
Images of outdoor scenes are degraded by absorption and scattering by the suspended particles and water droplets in the atmosphere. The light coming from a scene towards the camera is attenuated by fog and is blended with the airlight which adds more whiteness into the scene. Fog removal is highly desired in computer vision applications. Removing fog from images can significantly increase the visibility of the scene and is more visually pleasing. In this paper, we propose a method that can handle both homogeneous and heterogeneous fog which has been tested on several types of synthetic and real images. We formulate the restoration problem based on fusion strategy that combines two derived images from a single foggy image. One of the images is derived using contrast based method while the other is derived using statistical based approach. These derived images are then weighted by a specific weight map to restore the image. We have performed a qualitative and quantitative evaluation on 60 images. We use the mean square error and peak signal-to-noise ratio as the performance metrics to compare our technique with the state-of-the-art algorithms. The proposed technique is simple and shows comparable or even slightly better results with the state-of-the-art algorithms used for defogging a single image.
\end{abstract}

\section{KEYWORDS}

Airlight, Dark channel prior, Direct Attenuation, Fog removal, Image restoration

\section{INTRODUCTION}

Images of natural scenes are degraded due to bad weather such as fog, haze, mist, rain, smoke etc. The natural phenomena such as fog, smoke occurs mainly due to atmospheric absorption and scattering. While taking the image during bad weather condition, the radiance flux received by the camera from the scene point is attenuated along the line of sight. The incoming light is mixed with the light coming from all other directions called the airlight. In this phenomenon, the amount of scattering depends upon the distance of the scene points from the camera. Therefore there is a significant decay in the colour and the contrast of the captured image.

Fog removal is a fundamental requirement in the field of computer vision applications such as surveillance, remote sensing systems, outdoor object recognition and various camera-based intelligent driver assistance systems. Removal of fog from the input foggy image can

David C. Wyld et al. (Eds) : ACITY, DPPR, VLSI, WiMNET, AIAA, CNDC - 2015

pp. 115-123, 2015. @ CS \& IT-CSCP 2015

DOI : $10.5121 / \mathrm{csit} .2015 .51310$ 
exponentially increase the visibility of the scene. Recently, many computer vision algorithms suffer from low-contrast scene radiance. In many of the automatic systems, we assume that the input images have clear visibility. But it is not applicable in many of the real world situations. Fog is purely dependent upon unknown depth. Earlier fog removal techniques required multiple images of same scene under different environmental conditions. Such methods cannot be used for dynamic scenes and cannot be used on existing image sets. Therefore many researchers follow a new strategy, which is based on single gray-level or colour image without using any other extra source information. In this regard, several authors have proposed various defogging techniques such as dark channel prior [1], improved dark channel prior with different filters $[4,9,10]$, anisotropic diffusion [8] and Tarel's method [6,7]. Assessing the overall performance of the individual components in such systems is difficult, since the computational requirements and the fine tuning of the different parts become crucial. However, Tarel's method [7] is promising when applied to road images by taking into account that a large part of the image can be assumed to be a planar road whereas the dark channel prior method [1] is not dedicated to road images and thus the road part of the image is over enhanced. In this paper, we propose a technique that combines two derived images from a single foggy image. One of the images is derived using a contrast based method while the other is derived using a statistical based approach. These derived images are then weighted by a specific weight map to restore the image.

The remainder of this paper is organized as follows: Section 2 provides the mathematical model of a foggy image. Section 3 summarises the contrast and statistical based algorithms that have been widely used in the recent years to remove fog from single images. Section 4 explains the proposed fusion strategy in achieving fog removal. Section 5 describes the experimental setup and testing results which supports our claim. Finally, section 6 concludes this paper.

\section{BACKGROUND}

Fog is a combination of two components: Airlight and direct attenuation. Airlight adds whiteness into scene whereas the attenuation decreases the contrast in the scene as well as variation of scene colour which finally leads to a poor visual perception of the image. In computer vision the model widely used to describe the formation of a fog image can be expressed by the following equation:

$$
\text { Foggy Image }=\text { Direct Attenuation }+ \text { Airlight }
$$

The direct attenuation describes the scene radiance and its decay in the medium. It is a multiplicative distortion of the scene radiance.

$$
\text { Direct Attenuation }=\mathrm{J}(\mathrm{x}) \bullet \mathrm{t}(\mathrm{x})
$$

where $\mathrm{J}(\mathrm{x})$ is the scene radiance and $\mathrm{t}(\mathrm{x})$ is the medium transmission.

Airlight or atmospheric veil is caused due to scattering of light. Airlight is an additive one and is a function of the distance between camera and object.

$$
\text { Airlight }=\mathrm{A}(1-\mathrm{t}(\mathrm{x}))
$$

where $\mathrm{A}$ is the global atmospheric light. 
When the atmosphere is homogenous, the transmission $\mathrm{t}(\mathrm{x})$ can be expressed as:

$$
\mathrm{t}(\mathrm{x})=e^{-\beta \mathrm{d}(\mathrm{x})}
$$

where $\beta$ denotes the extinction coefficient of the atmosphere. It indicates that the scene radiance is attenuated exponentially with the scene depth d. Using equations (1), (2), (3) and (4) the intensity value I of $x$ th pixel a foggy image can be defined mathematically by equation (5):

$$
\mathrm{I}(\mathrm{x})=\mathrm{J}(\mathrm{x}) \bullet \mathrm{t}(\mathrm{x})+\mathrm{A}(1-\mathrm{t}(\mathrm{x}))
$$

The atmospheric light $\mathrm{A}$ is estimated as the maximum value of the corresponding region in the input (i.e., foggy) image.

\section{RELATED WORK}

Tarel and Hautiere [6] proposed a method for fast visibility restoration of images using a median filter algorithm. The restoration works with low complexity for gray and colour images, which adopts white balance, gamma correction, and tone mapping to maintain colour fidelity. Airlight is considered as a percentage between local standard deviation and local mean of the whiteness. Depth map is used to smooth along the corners. This method depends upon linear operations that require many parameters for the adjustment. Their method is not dedicated to road images and thus the road part of the image which is gray is over-enhanced due to the ambiguity between light coloured objects and the presence of fog. Furthermore, in some small edge regions, the desirable defogging results cannot be achieved.

In [7], an extended algorithm of [6] is proposed for better defogging of the roadway area. The method handles road images by taking into account that a large part of the image can be assumed to be a planar road. Thus the extended algorithm introduces a planar constraint to method [6]. The advantages of the improved version are its speed and small number of parameters. Authors claim that the algorithm produces similar quality results with homogeneous fog and it is able to better deal with the presence of heterogeneous fog.

He et al [1] proposed a method based upon dark channel prior which is basically used for single image defogging method. This dark channel prior is mainly used to measure the statistics of the outdoor fog-free image. The authors' method is based on the assumption that some pixels are having very low intensity in any one of the colour channel in the case of regions which do not cover the sky. These pixels are known as the dark pixels. In the case of foggy images, the intensity of the dark pixels is mainly contributed by the airlight. These dark pixels are used to estimate the fog transmission. The aim of the technique is to restore fog free image from the transmission map. Their method employs a dark channel prior which assumes every local patch $(15 \times 15)$ in the fog-free image have at least one colour component near zero. This assumption is sometime violated when there is no black body in some local patches. Instead of using a Markov random field, a soft matting algorithm is used to refine the transmission values. However, refining the observed transmission map with soft matting is computationally expensive. He et al [2] proposed a further refinement to the dark channel prior using a guided image filter in order to reduce the time complexity in [1]. Even though this method greatly reduces the time complexity, the original foggy image chosen as the reference image may lead to incomplete fog removal. 
However, the method in [7] is promising when applied to road images whereas the method in [1] is promising when applied to outdoor scene images such as cityscape and forests. Thus we propose a method for a single input image that can handle both road images and outdoor scene images by fusing the methods in [1] and [7] using an appropriate scaling factor. The proposed technique can handle both homogeneous and heterogeneous fog.

\section{METHOdOLOGY}

We formulate the restoration problem based on fusion strategy that combines two derived images, $C$ and $S$, from a single foggy image, I. Image $C$ is derived using contrast based method proposed in [7] while $S$ is derived using statistical based approach proposed in [1]. The overall framework of the fusion strategy is depicted in Figure 1.

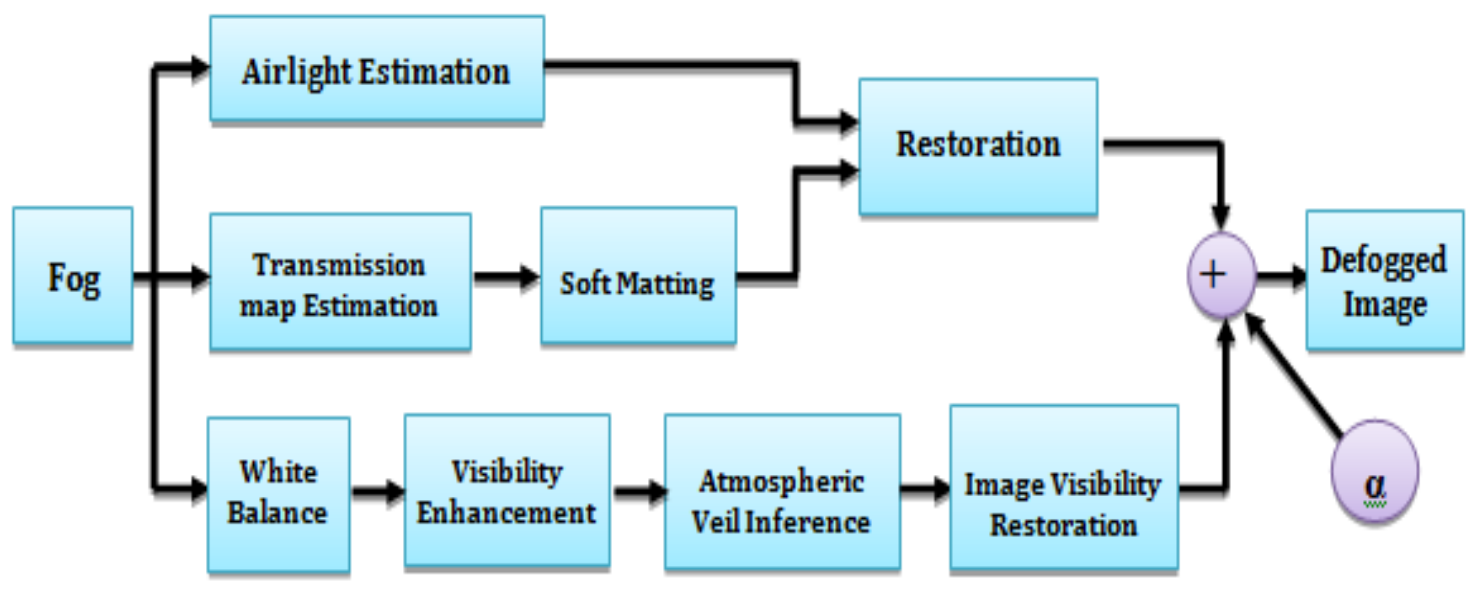

Fig 1: Flow diagram of the proposed fusion based single image defogging technique. The input images for the fusion strategy are obtained by following a statistical based approach on the foggy image as shown in the upper part of the flow diagram and in parallel by using a contrast based approach as shown in the lower part of the diagram. The obtained images are then weighted by a positive scaling factor $\alpha$ to produce defogged image.

\subsection{Statistical based approach}

To obtain the first input image $S$, a dark channel prior estimation is performed on the original image. It indicates that most local patches in fog-free outdoor images contain some pixels which have very low intensities in at least one colour channel. The atmospheric light is estimated from the most haze-opaque pixel. For example, the pixel with highest intensity is used as the atmospheric light [3]. The atmospheric light $\mathrm{A}$ is estimated by the average of the pixels in the original image that correspond to the top lightest $0.1 \%$ in the dark channel. Thus, A has three elements, the average values for each colour channel. Following the calculation of A, and assuming that the transmission in a local patch is constant, the transmission can be then estimated. For a refinement purpose, soft matting is applied to the initial transmission. Once we have the transmission map $t$ and the atmospheric light A, we can use (5) to recover the scene radiance $S$. 


\subsection{Contrast based approach}

In general, statistical based methods are not dedicated to road images and thus the roadway area in the defogged image is usually over-contrasted. The road can be reasonably assumed to be approximately flat. Thus in [7] the proposed method introduces a planar constraint to the method in [6] for better defogging of the roadway area. To obtain the second input image $C$, the first output is obtained by performing white balance operation on the original image. White balance is performed to correct the colour of the airlight prior to visibility restoration. When the white balance is correctly performed, the fog being pure white, this implies that A in (5) can be set to $(1,1,1)$, also assuming that the input image $\mathrm{I}$ is normalised between 0 and 1 . Following the white balance operation, a visibility enhancement algorithm is applied in order to avoid keeping certain amount of fog around outliers. The veil is inferred as a percentage of the difference between the local average and standard deviation of the whiteness within the observed image. This is achieved by using a median filter. The classical median filter preserves edges but not corners. This may induce artifacts on very structured scenes such as cityscapes and buildings. In order to preserve edges as well as corners with obtuse angle a median of median along lines filter is used [6]. The restoration of $C$ is performed by using the inferred atmospheric veil.

\subsection{Fusion strategy}

The images $C$ and $S$ obtained from the contrast based and statistical based methods respectively are then weighted by the following weight map:

$$
I=\frac{S+\alpha C}{1+\alpha}
$$

where $\alpha$ is a positive scalar.

Our approach to setting $\alpha$ is to take a subset of the synthetic images, compute the mean square error (MSE) between the fog-free and foggy images and set the scalar to the average sum of values of $\lceil 10 \sqrt{M S E}\rceil$. In general $\alpha=2$ yields better performance in defogging a single image. The proposed fusion strategy aims to preserve the regions with good visibility.

\section{EXPERIMENTAL SETUP}

\subsection{Dataset}

In general it is difficult to obtain the same background-foreground image with and without fog. In this work we make use of two image sets: synthetic and real images. We use images from the Foggy Road Image DAtabase (FRIDA) [7] for synthetic images and images from [1,5] for real images. The database in [7] consists of a total number of 66 colour images of different scenes such as city, highway and rural areas. Each image is of size $640 \times 480$. In this database four different types of fog: Uniform, variable sky intensity, variable fog density, and variable sky intensity and fog density were added to images. We have also tested real images from $[1,5]$ that are of cityscapes, buildings and forests. Each image is of size $600 \times 400$. A subset of images of road images and real images is shown in Figure 2(a). 


\subsection{Evaluation criteria}

In order to check the robustness of our proposed fusion based defogging approach the mean square error (MSE) and peak signal-to-noise ratio (PSNR) are estimated.

1) MSE: The mean squared error represents the cumulative squared error between the compressed and the original image. The lower the value of MSE, the lower the error. Given a noise-free $m \times n$ gray-level image I and its noisy approximation $K$, MSE is defined as:

$$
M S E=\frac{1}{m n} \sum_{i=0}^{m-1} \sum_{j=0}^{n-1}[I(i, j)-K(i, j)]^{2}
$$

2) PSNR: The Peak signal-to-noise ratio is computed in decibels between two images. Higher the PSNR, the better the quality of the compressed or reconstructed image. The PSNR is defined as:

$$
\begin{aligned}
\text { PSNR } & =10 \cdot \log _{10}\left(\frac{M A X_{I}^{2}}{M S E}\right) \\
& =20 \cdot \log _{10}\left(\frac{M A X_{I}}{\sqrt{M S E}}\right) \\
& =20 \cdot \log _{10}\left(M A X_{I}\right)-10 \cdot \log _{10}(M S E)
\end{aligned}
$$

where $M A X_{I}$ is the max intensity value in an image i.e., 1 in double.

\subsection{Testing Results}

We have performed a quantitative evaluation on 60 images taken from the database in [7] and few real images from [1,5] in which a qualitative analysis is performed. The rest of the six images from the database in [7] are used for estimating the novel parameter $\alpha$ of our approach. The $\alpha$ was varied from 1 to 6 by step size one. In each case of $\alpha$, MSE and PSNR were measured. In general our empirical results show that $\alpha=2$ yields better performance in defogging a single image. Therefore the results we report in Tables 1 and 2 are of $\alpha=2$.

Table 1 shows the quantized analysis of the mean square error for a subset of images and the overall MSE in the last row. As mean square error needs to be reduced the proposed algorithm shows better results than the available methods.

As PSNR need to be maximized the main goal is to increase the PSNR as much as possible. Table 2 clearly shows that PSNR is maximum for our proposed method. The method proposed in [7] clearly outperforms the method in [1] when considering MSE and PSNR as shown in the above tables. It has been observed that the proposed approach is comparable or slightly better than other two single image based technique in [1] and [7]. Figure 2 shows the qualitative comparison of the proposed method with methods in [1] and [7]. The restored images using our method are more natural and pleasing 
Table 1. COMPARISON OF MSE

\begin{tabular}{|c|c|c|c|}
\hline Image & $\begin{array}{c}\text { Contrast } \\
\text { based } \\
\text { method } \\
{[7]}\end{array}$ & $\begin{array}{c}\text { Statistical } \\
\text { based } \\
\text { method [1] }\end{array}$ & $\begin{array}{c}\text { Fusion } \\
\text { method } \\
\text { [Ours] }\end{array}$ \\
\hline 1 & 0.0114 & 0.0117 & 0.0112 \\
\hline$\vdots$ & $\vdots$ & $\vdots$ & $\vdots$ \\
\hline 10 & 0.0097 & 0.0098 & 0.0095 \\
\hline 11 & 0.0222 & 0.0226 & 0.0201 \\
\hline$\vdots$ & $\vdots$ & $\vdots$ & $\vdots$ \\
\hline 20 & 0.0134 & 0.0138 & 0.0131 \\
\hline 21 & 0.0175 & 0.0188 & 0.0173 \\
\hline$\vdots$ & $\vdots$ & $\vdots$ & $\vdots$ \\
\hline 30 & 0.0206 & 0.0207 & 0.0204 \\
\hline 31 & 0.0162 & 0.0181 & 0.0161 \\
\hline$\vdots$ & $\vdots$ & $\vdots$ & $\vdots$ \\
\hline 40 & 0.0148 & 0.0157 & 0.0145 \\
\hline 41 & 0.0199 & 0.0194 & 0.0192 \\
\hline$\vdots$ & $\vdots$ & $\vdots$ & $\vdots$ \\
\hline 50 & 0.0217 & 0.0221 & 0.0213 \\
\hline 51 & 0.0218 & 0.0221 & 0.0215 \\
\hline$\vdots$ & $\vdots$ & $\vdots$ & $\vdots$ \\
\hline 60 & 0.0203 & 0.0178 & 0.0192 \\
\hline Average & 0.0171 & 0.0182 & $\mathbf{0 . 0 1 6 8}$ \\
\hline & & & \\
\hline
\end{tabular}
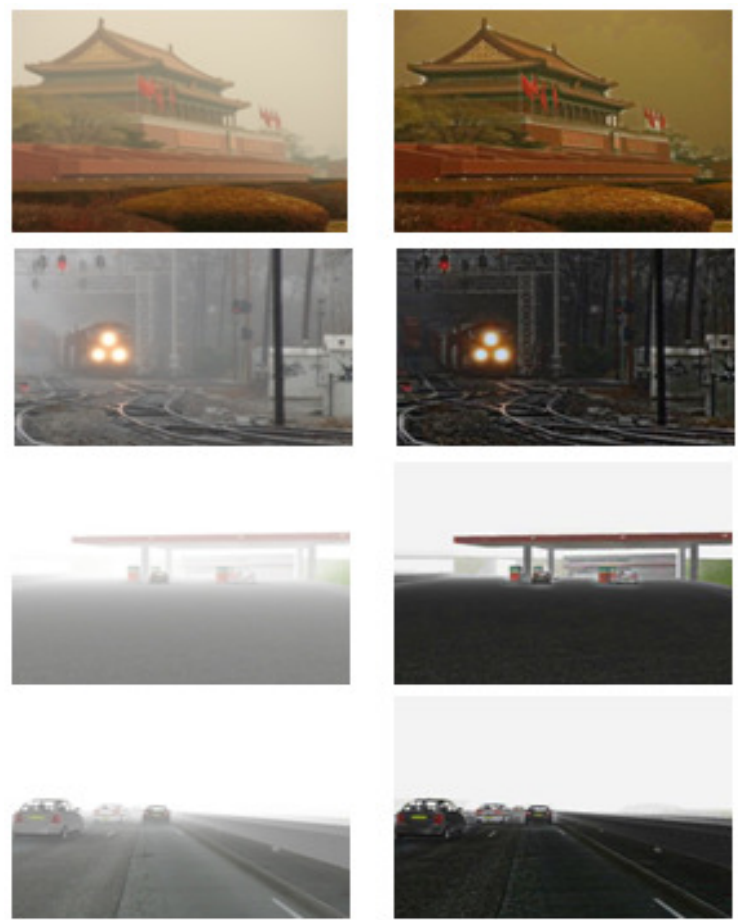

(a)
Table 2. COMPARISON OF PSNR

\begin{tabular}{|c|c|c|c|}
\hline Image & $\begin{array}{c}\text { Contrast } \\
\text { based } \\
\text { method [7] }\end{array}$ & $\begin{array}{c}\text { Statistical } \\
\text { based } \\
\text { method [1] }\end{array}$ & $\begin{array}{c}\text { Fusion } \\
\text { method } \\
\text { [Ours] }\end{array}$ \\
\hline 1 & 67.5509 & 67.4545 & 67.6467 \\
\hline$\vdots$ & $\vdots$ & $\vdots$ & $\vdots$ \\
\hline 10 & 68.2442 & 68.2119 & 68.3646 \\
\hline 11 & 64.6625 & 64.5966 & 65.1051 \\
\hline$\vdots$ & $\vdots$ & $\vdots$ & $\vdots$ \\
\hline 20 & 66.3743 & 66.2571 & 66.51 \\
\hline 21 & 66.3174 & 66.0871 & 66.366 \\
\hline$\vdots$ & $\vdots$ & $\vdots$ & $\vdots$ \\
\hline 30 & 64.9967 & 64.9789 & 65.0451 \\
\hline 31 & 65.4234 & 65.5383 & 65.5417 \\
\hline$\vdots$ & $\vdots$ & $\vdots$ & $\vdots$ \\
\hline 40 & 66.416 & 66.1842 & 66.5158 \\
\hline 41 & 67.0691 & 66.3794 & 67.2119 \\
\hline$\vdots$ & $\vdots$ & $\vdots$ & $\vdots$ \\
\hline 50 & 64.7735 & 64.6943 & 64.8412 \\
\hline 51 & 64.7442 & 64.6869 & 64.8161 \\
\hline$\vdots$ & $\vdots$ & $\vdots$ & $\vdots$ \\
\hline 60 & 66.6173 & 66.0919 & 66.6434 \\
\hline Average & 66.1385 & 65.7917 & 66.2123 \\
\hline & & & \\
\hline
\end{tabular}
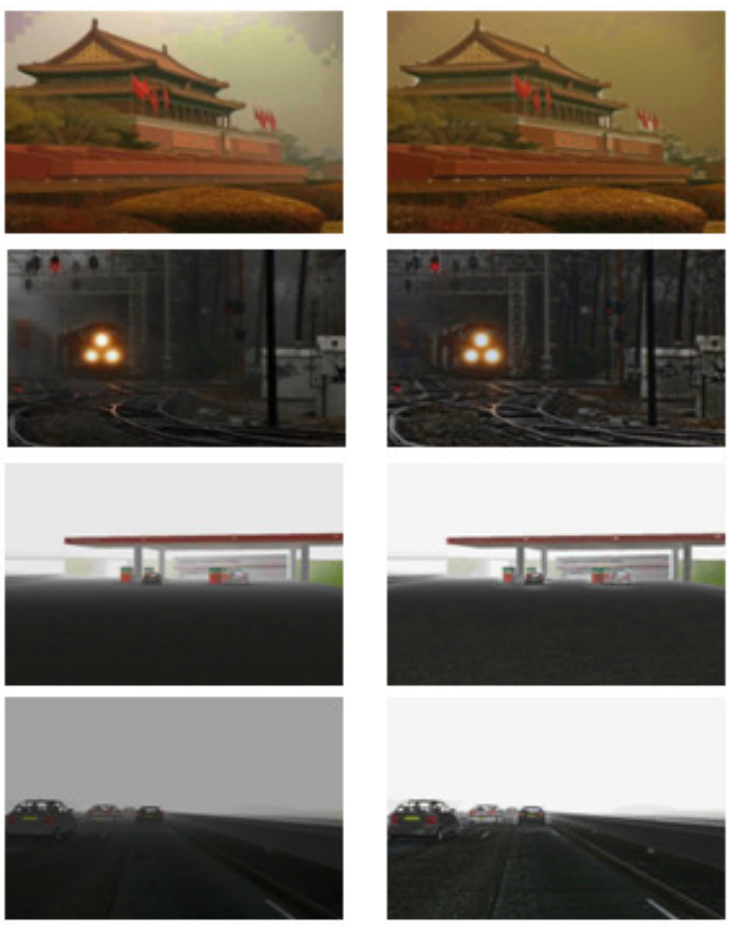

(c)

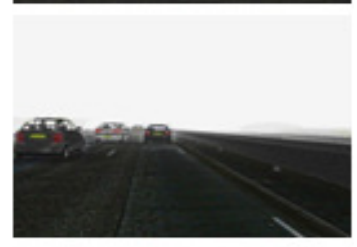

(d)

Fig. 2: Comparison of contrast and statistical based techniques with our fusion based strategy. (a) Input image: First two rows are of real images whereas the last two rows are of road images (b) Tarel's results (c) He's results (d) Our results. 


\section{CONCLUSION}

In this paper, a simple but efficient method is proposed to improve single image defogging based on a fusion strategy, which can work well for synthetic (i.e., road images) and real images. The fusion based defogging approach can effectively restore image colour balance and remove fog. This technique is based on selection of appropriate weight map to fuse contrast and statistical based approaches in single image defogging applications. Moreover, it has been observed that this approach outperform the other single image based defogging techniques. The restored images are more natural and pleasing. It has been proved that our method has strong robustness and high availability which can be widely applied to colour images. The proposed method is faster and yields accurate results.

\section{REFERENCES}

[1] K. He, J. Sun, and X. Tang, "Single image haze removal using dark channel prior", In proceedings of IEEE International Conference on Computer Vision and Pattern Recognition, pp. 1956-1963, 2009.

[2] K. He, J. Sun and X. Tang, "Guided image filtering", In proceedings of the Eleventh IEEE European Conference on Computer Vision, pp. 1-14, 2010.

[3] S.G. Narasimhan and S.K. Nayar, "Contrast restoration of weather degraded images", In IEEE Transactions on Pattern Analysis and Machine Intelligence, vol. 25, pp. 713-724, 2003.

[4] J. Pang, A. Oscar, and G. Zheng, "Improved single image dehazing using guided filter", In proceedings of the APSIPA Annual Summit and Conference, ACM, pp.1-4, 2011.

[5] Real images: https://sites.google.com/site/computervisionadinastoica/final-project

[6] J. P. Tarel and N. Hautiere, "Fast visibility restoration from a single color or gray level image", In proceedings of IEEE International Conference on Computer Vision, pp. 2201-2208, 2009.

[7] J.P. Tarel, N. Hautiere, A. Cord, D. Gruyer, and H. Halmaoui, "Improved visibility of road scene images under heterogeneous fog", In proceedings of IEEE Intelligent Vehicle Symposium, pp. 478$485,2010$.

[8] A.K. Tripathi and S. Mukhopadhyay, "Single image fog removal using anisotropic diffusion", In proceedings of IET Image Process, vol. 6, no. 7, pp. 966-975, 2012.

[9] S. Yanjuan, R. Liu and W. He, "Image Haze Removal of Wiener Filtering Based on Dark Channel Prior", In proceedings of Eighth IEEE International Conference on Computational Intelligence and Security, pp. 318-322, 2012.

[10] Yan Wang and Bo Wu, "Improved Single Image Dehazing using Dark Channel Prior", In proceedings of IEEE Conference on Intelligent Computing and Intelligent Systems, vol. 2, pp. 789 $792,2010$. 


\section{AUTHORS}

V. Thulasika is an Associate Software Engineer at WSO2 Solutions, Sri Lanka. She received her BSc Honours in Computer Science (2015) from the University of Jaffna, Sri Lanka. Her research interest includes computational photography and image processing.

A. Ramanan is a Senior Lecturer at the Department of Computer Science, University of Jaffna. He received his BSc Honours in Computer Science (2002) from the University of Jaffna, Sri Lanka and his PhD from the University of Southampton, UK (2010). His research interests include computer vision and machine learning.
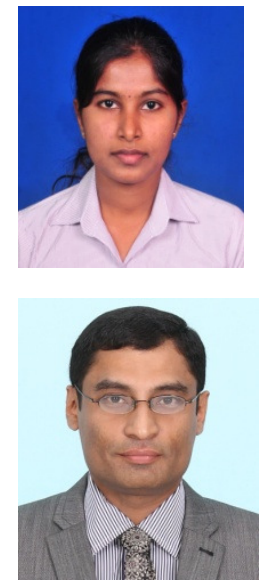